\title{
Inaccuracy of ICD-9 Codes for Chronic Kidney Disease: A Study from Two Practice-based Research Networks (PBRNs)
}

\author{
Charlotte W. Cipparone, BA, Matthew Withiam-Leitch, MD, PhD, \\ Kim S. Kimminau, PhD, Chet H. Fox, MD, Ranjit Singh, MD, MBBChir, MBA, \\ and Linda Kabn, PbD
}

Background: Inaccurate use of International Classification of Diseases, Ninth Revision (ICD-9), codes obfuscates registries used for research, resulting in unreliable data and inaccurate measurement of outcomes, and it may contribute to mismanagement of patients. Thus it is important to understand the prevalence of ICD-9 code misuse. We chose chronic kidney disease (CKD) as a condition of interest after several patients recruited for a previous study indicated they did not have the disease, despite the presence of the ICD-9 code (585.x) in their electronic medical record (EMR).

Methods: Retrospective chart review of patients with the ICD-9 code for CKD stage $3(585.3 ; n=$ 325). Data were collected from EMRs at 3 primary care practices Buffalo, New York $(n=2)$, and Kansas City, Kansas $(\mathrm{n}=1)$.

Results: Across all practices, $47 \%$ of patients with the CKD ICD-9 code did not have clinical indicators for the disease, based on Kidney Disease Outcomes Quality Initiative guidelines.

Conclusions: The CKD stage 3 ICD-9 code usage did not accurately reflect the prevalence of disease among this population. This has clinical implications because patients may be treated or receive tests for a disease they do not have. This also presents an important issue for research projects that rely on accurate data from EMRs to identify and recruit patients. (J Am Board Fam Med 2015;28:678-682.)

Keywords: Chronic Disease, Chronic Kidney Diseases, Clinical Coding, Diagnostic Errors, Electronic Medical Records, Medical Errors

\footnotetext{
This article was externally peer reviewed.

Submitted 30 April 2014; revised 29 December 2014; accepted 12 January 2015.

From the Primary Care Research Institute, Department of Family Medicine, University at Buffalo, Buffalo, NY (CWC, MW-L, CHF, RS, LK); and the Department of Family Medicine, University of Kansas Medical Center, Kansas City, KS (KSK).

Funding: This study was supported by an American Academy of Family Physicians Research Stimulation Grant (no. G1203RS to LK and CHF); the National Institute of Diabetes and Digestive and Kidney Diseases (grant no. 1R01DK090407-01A1; www.clinicaltrials.gov identifier NCT01767883); and the Department of Health and Human Services, Health Resources and Services Administration Pre-Doctoral Training in Primary Care Grant (award no. 5 D56HP23273-03-00, September 30, 2011 through September 29, 2016).

Conflict of interest: none declared.

Corresponding author: Charlotte W. Cipparone, BA, Primary Care Research Institute, Department of Family Medicine, University at Buffalo, 77 Goodell St, Suite 220, Buffalo, NY 14203 (E-mail: charlotte.cipparone@gmail.com).
}

Electronic databases are increasingly being used to track and analyze population health outcomes. ${ }^{1}$ The ability to track disease longitudinally among large populations is an important innovation for health research and public health. It is imperative, however, to ensure that the International Classification of Diseases, Ninth Revision (ICD-9), codes are reliably accurate. ${ }^{2,3}$

The issue of ICD-9 code accuracy was raised when we developed patient registries using these codes to identify patients with chronic kidney disease (CKD) for an institutional review board-approved qualitative interview study. The study was conducted in a practice in an Upstate New York practice-based research network (UNYNET). Patients identified in this registry were sent a letter inviting them to participate in the study. Several patients responded with alarm, indicating that they did not have CKD. A subsequent manual chart 
Table 1. Demographics of Chronic Kidney Disease (CKD) Stage 3 Patients at Three Residency Teaching Sites

\begin{tabular}{|c|c|c|c|c|}
\hline Characteristic & $\begin{array}{l}\text { Total Sample } \\
(\mathrm{n}=325)\end{array}$ & $\begin{array}{l}\text { Buffalo Practice } 1 \\
\quad(\mathrm{n}=109)\end{array}$ & $\begin{array}{l}\text { Buffalo Practice } 2 \\
\quad(\mathrm{n}=95)\end{array}$ & $\begin{array}{c}\text { Kansas City Practice } \\
\qquad(\mathrm{n}=121)\end{array}$ \\
\hline Female sex & $56(182)$ & $61(67)$ & $44(42)$ & $60(73)$ \\
\hline \multicolumn{5}{|l|}{ Age (years) } \\
\hline Mean (SD) & $66.8(13.5)$ & $67.8(13.4)$ & $63(12.8)$ & $69.1(13.5)$ \\
\hline Range & $32-96$ & $32-96$ & $35-93$ & $34-95^{*}$ \\
\hline \multicolumn{5}{|l|}{ Race/ethnicity } \\
\hline Hispanic (any race) & $3(11)$ & $5(5)$ & $0(0)$ & $5(6)$ \\
\hline Black & $68(222)$ & $83(90)$ & $86(82)$ & $41(50)$ \\
\hline White & $25(82)$ & $10(11)$ & $14(13)$ & $56(58)$ \\
\hline American Indian/Alaska Native & $0(1)$ & $0(0)$ & $0(0)$ & $0.8(1)$ \\
\hline Asian/unknown/other/decline to answer & $5(15)$ & $3(3)$ & $0(0)$ & $10(12)$ \\
\hline
\end{tabular}

Data are \% (n) unless otherwise indicated.

*Data from one patient are missing.

$\mathrm{SD}$, standard deviation.

review verified that several patients did not meet national guideline-based CKD criteria, although their chart contained the ICD-9 code for the disease. We then expanded the study to another UNYNET practice and a comparable practice in the Kansas Physicians Engaged in Prevention Research (KPEPR) network to determine whether the results were unique to our original practice.

ICD-9 coding inaccuracies raise concerns for both clinical treatment and research. Patients with an ICD code for a disease they do not have may undergo unnecessary treatment or tests. In addition, subjects in research studies recruited from registries based on ICD codes may not have the disease in question, compromising the reliability of the findings. ${ }^{4-6} \mathrm{Al}-$ though the literature on the accuracy of electronic health records is limited, recent studies suggest that data in electronic health records may not be appropriate for quality reporting. ${ }^{6}$

The objectives of this study were to (1) determine the prevalence of misdiagnosis among patients with stage 3 CKD at the original UNYNET practice site, and (2) to compare this prevalence to that within a similar UNYNET practice and a KPEPR practice.

\section{Methods}

This study entailed a collaboration between 2 PBRNs. We conducted retrospective chart reviews of patients from 2 primary care practices in Buffalo, New York (UNYNET sites) and 1 primary care practice in Kansas City, Kansas (KPEPR site). All 3 sites are residency teaching sites located in low- income neighborhoods and use a certified electronic medical record (EMR). Two sites treat predominantly African American patients (Table 1).

Patients selected for chart review were adults (>18 years old) with the ICD-9 code 585.3 (CKD stage 3 ) recorded in their EMR. A registry of these patients was pulled from each practice and given to the research team for analysis.

The Buffalo and Kansas teams used a single chart review protocol to guide data collection (Table 2). The protocol was informed by the Kidney Disease Outcomes Quality Initiative (KDOQI) guidelines, which state that a patient meets criteria for CKD stage 3 if they have had 2 estimated glomerular filtration rates $<60$ at least 90 days apart or have had 2 albumin creatinine ratios $>30$ at least 90 days apart. This study was approved the institutional review boards of both the University at Buffalo and the University of Kansas Medical Center.

\section{Results}

Comorbidities and demographics were similar across practices (Table 1). Among the 3 practices, $47 \%$ of the patients did not have sufficient evidence recorded in their chart to support the diagnosis of CKD. Similar prevalence of misdiagnosis was found at each practice (Table 3). At the 2 Buffalo sites, test results recorded in the charts were analyzed further (Table 4).

\section{Discussion}

Based on our findings, nearly half of patients in the registries did not have the clinical indicators to 
Table 2. Chart Review Protocol

\begin{tabular}{|c|c|}
\hline ICD-9 Code & 585.3 \\
\hline Race/ethnicity & $\begin{array}{c}\text { White, African } \\
\text { American, } \\
\text { Hispanic, } \\
\text { or Native } \\
\text { American }\end{array}$ \\
\hline Age & Numeric value \\
\hline Body mass index & Numeric value \\
\hline $\begin{array}{l}\text { Two most recent GFR values, } \\
\text { with dates }\end{array}$ & Numeric value* \\
\hline $\begin{array}{l}\text { Two most recent ACR values, } \\
\text { with dates }\end{array}$ & Numeric value* \\
\hline Diagnosis based on GFR correct? & Yes/No \\
\hline Diagnosis based on ACR correct? & Yes/No \\
\hline ACR date & Date \\
\hline $\begin{array}{l}\text { ACR verified? (2 successive ACRs } \\
\text { at least } 90 \text { days apart) }\end{array}$ & $\begin{array}{l}\text { Dates and } \\
\text { values of } \\
\text { the } 2 \\
\text { ACRs }\end{array}$ \\
\hline \multicolumn{2}{|l|}{ Comorbidities } \\
\hline Diabetes mellitus & Yes/no \\
\hline Hypertension & Yes/no \\
\hline Congestive heart failure & Yes/no \\
\hline Sleep apnea & Yes/no \\
\hline Acute kidney injury & Yes/no \\
\hline Coronary artery disease & Yes/no \\
\hline
\end{tabular}

*Values were recorded only at the Buffalo sites. ACR, albumin-to-creatinine ratio; GFR, glomerular filtration rate; ICD-9, International Classification of Diseases, Ninth Revision.

support a diagnosis of CKD stage 3, suggesting that registries developed from ICD-9 codes may not accurately represent the prevalence of CKD stage 3 in these practices.

The reasons behind this high prevalence of misdiagnosis cannot be defined by this study. However, this finding raised many questions as to why this may be occurring. Some possible reasons include CKD-specific issues, alternative uses of ICD-9 codes, and challenges inherent in the practices where this study was conducted. These issues are discussed below.

Table 3. Prevalence of Misdiagnosis

\begin{tabular}{lcc}
\hline Site & Patients, n & $\begin{array}{c}\text { Prevalence of } \\
\text { Misdiagnosis, } \\
\text { n (\%) }\end{array}$ \\
\hline Buffalo practice 1 & 109 & $48(44)$ \\
Buffalo practice 2 & 95 & $52(54)$ \\
Kansas City practice & 121 & $54(45)$ \\
Total & 325 & $154(47)$ \\
\hline
\end{tabular}

Table 4. Breakdown of Misdiagnosed Patients*

\begin{tabular}{lcccc}
\hline & \multicolumn{3}{c}{ Patients (n) } & \\
\cline { 2 - 4 } & $\begin{array}{c}\text { Buffalo } \\
\text { Practice } \\
1(48)\end{array}$ & $\begin{array}{c}\text { Buffalo } \\
\text { Practice } \\
2(52)\end{array}$ & $\begin{array}{c}\text { Total } \\
(100)\end{array}$ & $\begin{array}{c}\text { Prevalence } \\
(\%)\end{array}$ \\
\hline 1 Normal, 1 & 19 & 18 & 37 & 37 \\
$\quad$ abnormal GFR & & & & \\
1 Normal GFR & 3 & 8 & 11 & 11 \\
1 Abnormal GFR & 6 & 6 & 12 & 12 \\
2 Normal GFRs & 19 & 14 & 33 & 33 \\
No recorded GFRs & 1 & 6 & 7 & 7 \\
No recorded ACRs & 42 & 18 & 60 & 60 \\
\hline
\end{tabular}

*Data are from the Buffalo sites only.

ACR, albumin-to-creatinine ratio; GFR, glomerular filtration rate.

Studies have suggested that primary care physicians are uncomfortable with or unaware of the KDOQI guidelines for CKD, especially with patients in the early to moderate stages of the disease. $^{7-9}$ KDOQI guidelines state that a patient meets criteria for CKD stage 3 if they have had 2 estimated glomerular filtration rates $<60$ at least 90 days apart or have had 2 albumin creatinine ratios $>30$ at least 90 days apart. ${ }^{10,11}$ Thus, accurate diagnosis relies on 2 abnormal lab tests observed in the appropriate time frame. However, our findings suggest that physicians may be diagnosing CKD after only 1 abnormal test result. Furthermore, many of the misdiagnosed patients had normal or insufficient test results, suggesting that physicians may be diagnosing based on factors not supported by the KDOQI guidelines ${ }^{12}$ (see Table 4).

Another explanation for our finding involves the use of ICD-9 codes in ways other than to signify a diagnosis. ICD-9 codes may be used by practitioners to signify a "working diagnosis," to allow a test or procedure to be covered by a patient's insurance or to increase reimbursement for the practice. ${ }^{1,13}$ Billing staff may add or modify codes for the same reasons.

This high prevalence of CKD misdiagnosis that we observed might be related to the characteristics of the practices where we conducted chart reviews. All 3 practices are residency teaching sites in family medicine. Residents may be even less comfortable with $\mathrm{CKD}$ guidelines than attending physicians, leading to more inaccurate diagnoses. ${ }^{7}$ In addition, residency practices experience less continuity of care because of student turnover. Reduced continuity of care could contribute to the perpetuation 
of an inaccurate diagnosis in the EMR because physicians may be unlikely to question a diagnosis made by a previous provider. These practices also are located in low-income neighborhoods where physicians treat patients with complex medical and social problems. These competing demands could potentially distract physicians from guideline-concordant CKD diagnosis for patients in the early stages of the disease.

Although we cannot yet determine the reasons behind this issue, it has important implications for both research and patient care. Electronic databases built from registries of patients with certain diagnoses, as tracked by ICD-9 codes, are increasingly used for research. ${ }^{4,13}$ If a large percentage of these ICD-9 codes, such as the ones found in this study, do not reflect the actual presence or prevalence of disease, the research findings will be skewed. Outcomes research may be tainted and irreproducible if the patients identified in these registries do not have the disease in question. ${ }^{4}$

In addition, if the prevalence of misdiagnosis we observed in this patient cohort is present in other populations, thousands of patients may be mismanaged. This creates an enormous patient safety problem, since patients may be receiving unnecessary tests and/or treatments. For example, a patient treated with an angiotensin-converting enzyme/angiotensin receptor blocker for an erroneous diagnosis of CKD may be unnecessarily exposed to the risk of hyperkalemia, a serious adverse event.

This study has several limitations. First, we reviewed only charts of patients who already had a diagnosis of CKD stage 3. Our study did not investigate patients who met criteria for CKD but did not have a corresponding ICD-9 code in their records. The under-recognition of CKD is a welldocumented problem, which is why were surprised to find evidence of overdiagnosis among this population. ${ }^{8}$ The clinical data from a limited population $(n=325)$ makes it difficult to determine the reasons behind our findings. A strength of this study, however, was that we were able to find similar results among 3 separate practices in 2 different regions of the country. The practices we chose had similar patient populations, and all 3 were residency teaching clinics. We believe this is a strength of our study. Our findings suggest, however, that these results might be replicated in practices similar to those we studied. This would implicate thousands of misdiagnosed patients. More research needs to be done to determine whether these findings are reproducible in a broader range of primary care practices. If this is confirmed, practices similar to those we studied may be affected. Further research should be undertaken to uncover the reasons behind the high prevalence of misdiagnosis, and initiatives should be developed to address this issue.

\section{Conclusion}

Nearly half of the patients diagnosed with CKD stage 3 in these 3 primary care practices did not meet diagnostic criteria based on national, evidence-based guidelines. Although further research needs to be done to determine the underlying causes of this finding, it presents serious implications for both research and clinical practice in an environment increasingly reliant on reliable electronic data.

The authors acknowledge Joel Hake, MD; Bethany Duff, MD; and Jana Zaudke, MD for their contributions to the data collection and analysis.

\section{References}

1. O'Malley KJ, Cook KF, Price MD, Wildes KR, Hurdle JF, Ashton CM. Measuring diagnoses: ICD code accuracy. Health Serv Res 2005;40:1620-39.

2. Singh H, Giardina TD, Forjuoh SN, et al. Electronic health record-based surveillance of diagnostic errors in primary care. BMJ Qual Saf 2011;21:93100.

3. Kudyakov R, Bowen J, Ewen E, et al. Electronic health record use to classify patients with newly diagnosed versus preexisting type 2 diabetes: infrastructure for comparative effectiveness research and population health management. Popul Health Manag 2012;15:3-11.

4. Weiskopf NG, Weng C. Methods and dimensions of electronic health record data quality assessment: enabling reuse for clinical research. J Am Med Inform Assoc 2012;20:144-51.

5. Singh H, Meyer AN, Thomas EJ. The frequency of errors in outpatient care: estimation from three large observational studies involving US populations. BMJ Qual Saf 2014; 23:727-31.

6. Chan KS, Fowles JB, Weiner JP. Review: electronic health records and the reliability and validity of quality measures: a review of the literature. Med Care Res Rev 2010;67:503-27.

7. Agrawal V, Ghosh AK, Barnes MA, McCullough PA. Awareness and knowledge of clinical practice guidelines for CKD among internal medicine residents: a national online survey. Am J Kidney Dis 2008;52:1061-9. 
8. Fox CH, Brooks A, Zayas LE, McClellan W, Murray B. Primary care physicians' knowledge and practice patterns in the treatment of chronic kidney disease: an Upstate New York Practice-based Research Network (UNYNET) study. J Am Board Fam Med 2006; 19:54-61.

9. Plantinga LC, Tuot DS, Powe NR. Awareness of chronic kidney disease among patients and providers. Adv Chronic Kidney Dis 2010;17:225-36.

10. Fox CH. A decade after the KDOQI CKD guidelines: impact on primary care. Am J Kidney Dis 2012;60:707-9.
11. National Kidney Foundation. K/DOQI clinical practice guidelines for chronic kidney disease: evaluation, classification, and stratification. Am J Kidney Dis 2002;29(2 Suppl 1):S1-266.

12. Levey AS, de Jong PE, Coresh J, et al. The definition, classification, and prognosis of chronic kidney disease: a KDIGO Controversies Conference report. Kidney Int 2010;80:17-28.

13. Schneeweiss S, Avorn J. A review of uses of health care utilization databases for epidemiologic research on therapeutics. J Clin Epidemiol 2005;58:323-37. 